\title{
Why We Should Use High Values for the Smoothing Parameter of the Hodrick-Prescott Filter
}

\author{
Gebhard Flaig* \\ University of Munich
}

JEL C22; C52

Hodrick-Prescott filter; Wiener-Kolmogorov filter; smoothing parameter; trends; cycles.

Received: 22.10 .2013

Revision received: 05.06.2014

Accepted: 03.08.2014

\section{Summary}

The HP filter is the most popular filter for extracting the unobserved trend and cycle components from a time series. Many researchers consider the smoothing parameter $\lambda=1600$ as something like a universal constant. It is well known that the HP filter is an optimal filter under some restrictive assumptions, especially that the "cycle" is white noise. In this paper we show that we can get a good approximation of the optimal Wiener-Kolmogorov filter for autocorrelated cycle components by using the HP filter with a much higher smoothing parameter than commonly used. In addition, a new method - based on the properties of the differences of the estimated trend - is proposed for the selection of the smoothing parameter.

\section{Introduction}

The probably most popular filter for extracting a trend and a cycle component from an observed time series is the Hodrick-Prescott filter (Hodrick/Prescott 1997). The features of this filter were intensively studied in the literature (see, among many others, King/Rebelo 1993, Harvey/Jaeger 1993, Cogley/Nason 1994, Kaiser/Maravall 2001). The properties of the HP filter in the time and frequency domain depend mainly on a smoothing parameter $\lambda$ which governs the smoothness of the estimated trend and the shape of the estimated cycle. In most empirical applications a value of 1600 is used for $\lambda$. This is the value recommended by Hodrick and Prescott (1997) for quarterly data. They motivate this value by the assumption that the ratio of the variance of the cyclical component to the variance of the second differences of the trend (the inverse signal-to-noise ratio) of US GDP is about 1600 .

It is well known that the HP filter with a smoothing parameter $\lambda$ is optimal (in the sense that the mean square error (MSE) of the estimated components is minimal) if the second differences of the trend follow a white noise process (the trend is integrated of order two), the cyclical component is white noise as well and $\lambda$ is set to the inverse signal-to-noise

* The author thanks Andreas Blöchl, seminar participants at the Statistische Woche in Vienna and at the ifo lunchtime seminar, the editor and two referees for useful suggestions. 
ratio. These assumptions are clearly not appropriate in many applications. For instance, when the trend is a random walk (with constant drift), its second differences follow a noninvertible MA(1), not a white noise process. Most economists would argue that the cycle is also not white noise but should be specified as an autocorrelated stationary process. In all these cases, the suggestion of Hodrick and Prescott has no sound justification and the HP filter is a pure ad-hoc procedure with possibly dubious features.

An alternative to ad-hoc filters like the HP filter is the specification and estimation of unobserved components models (Harvey 1989). The Kalman filter and smoother deliver in this case the optimal filter weights which are identical to those of the classical WienerKolmogorov approach (Gomez 1999). A second possibility is to estimate an ARIMA model and apply the Beveridge-Nelson decomposition (Beveridge/Nelson 1981) or the "canonical" decomposition (Box et al. 1978).

Despite those theoretically attractive alternatives and many disadvantages and problems of the HP filter (for a survey and some new results concerning the use of HP filtered series in econometric models see Meyer/Winker 2005) the Hodrick-Prescott filter is widely used in academic research and practical business cycle analysis. One reason may be that the use of the HP filter does not require the specification and estimation of possibly complex time series models. It is a very simple and fast procedure. In the following we do not present a further investigation concerning the pro's and con's of the the HP filter but simply accept the fact that the HP filter is the probably most used approach in economics for the extraction of trend and cycle components of macroeconomic data.

The focus in this paper is on the choice of a reasonable value for the smoothing parameter of the HP filter. The reference approach is the Wiener-Kolmogorov filter which minimizes the mean square error of generated unobserved components like the trend or the cycle. Assuming a doubly infinite time series we derive for different specifications of the trend and the cycle components the optimal Wiener-Kolmogorov filter and search for that $\lambda$ for which the distance between the gain functions of the Wiener-Kolmogorov and of the Hodrick-Prescott filter is minimal. The results imply that in most realistic settings we should use much higher values for the smoothing parameter than the inverse signal-tonoise ratio (as suggested by Hodrick/Prescott 1997). For instance, assuming for the cycle an $\operatorname{AR}(1)$ model with a parameter of 0.7 , the optimal $\lambda$ is about five times higher than the inverse signal-to-noise ratio. The theoretical results are corroborated by a simulation study for time series with 160 observations. It is shown that by choosing a higher value for $\lambda$ than the inverse signal-to-noise ratio one can achieve a remarkable efficiency gain.

The obtained results are useful but not directly applicable in practice as we don't know the true signal-to-noise ratio. In the second part of the paper we derive a simple rule for the determination of a reasonable value for $\lambda$. The basic idea is that the first and/or second differences of the extracted trend should not exhibit a cyclical behavior. We propose to use the HP filter with different values of $\lambda$ and select the minimum value for which the first and second differences of the generated trend show no cyclical behavior. This choice can be based on visual inspection or on a more formal analysis in the time or frequency domain.

The paper is organized as follows: Section 2 outlines the model, the optimal WienerKolmogorov filter and the Hodrick-Prescott filter. In section 3 we derive the optimal smoothing parameter for the case of autocorrelated cycles. In section 4 we discuss a new suggestion for selecting $\lambda$. Section 5 reports the results for an empirical application and section 6 concludes. 


\section{Theoretical framework}

\subsection{The model}

We specify a time series $\left\{y_{t}\right\}$ as the sum of a non-stationary trend component $\left\{\mu_{t}\right\}$ and a stationary cycle $\left\{c_{t}\right\}$

$$
y_{t}=\mu_{t}+c_{t} \text {. }
$$

The trend component is modeled as a $\mathrm{n}$-fold integrated variable

$$
(1-L)^{n} \mu_{t}=\eta_{t},
$$

where $n$ is a positive integer and $\eta_{t}$ is white noise with $E \eta_{t}=0$ and $\operatorname{Var} \eta_{t}=\sigma_{\eta}^{2}$.

The cycle is specified as a stationary $\operatorname{AR}(2)$ process (with $\operatorname{AR}(1)$ as a special case)

$$
c_{t}=\varphi_{1} c_{t-1}+\varphi_{2} c_{t-2}+\epsilon_{t}
$$

or

$$
\Phi(L) c_{t}=\epsilon_{t}
$$

where $\Phi(L)=1-\varphi_{1} L-\varphi_{2} L^{2}$ and $\epsilon_{t}$ is white noise with $E \epsilon_{t}=0$ and $\operatorname{Var} \epsilon_{t}=\sigma_{\epsilon}^{2}$. It is further assumed that $\eta_{t}$ and $\epsilon_{t}$ are uncorrelated at all leads and lags. $\operatorname{AR}(1)$ and $\operatorname{AR}(2)$ models are frequently used for modeling business cycles. The approach could be extended to cases where the cycle follows a general stationary and invertible $\operatorname{ARMA}(\mathrm{p}, \mathrm{q})$ process. In the following we derive some properties of the components and the observed time series in the frequency domain. The frequency domain properties are the basis for the applications in section 3 . The specification of the trend and cycle components implies the following model for the observed time series

$$
y_{t}=(1-L)^{-n} \eta_{t}+\Phi(L)^{-1} \epsilon_{t} .
$$

The stationary form we can express as

$$
(1-L)^{n} y_{t}=\eta_{t}+\frac{(1-L)^{n}}{\Phi(L)} \epsilon_{t}
$$

The spectral density of the cycle is given by (Sargent 1987: 262)

$$
\begin{aligned}
g_{c}(\omega) & =\left[1+\varphi_{1}^{2}+\varphi_{2}^{2}-2 \varphi_{1}\left(1-\varphi_{2}\right) \cos \omega-2 \varphi_{2} \cos 2 \omega\right]^{-1} \sigma_{\epsilon}^{2} / 2 \pi \\
& =\left[1+\varphi_{1}^{2}+\varphi_{2}^{2}-2 \varphi_{1}\left(1-\varphi_{2}\right) \cos \omega-2 \varphi_{2} \cos 2 \omega\right]^{-1} \frac{\left(1+\varphi_{2}\right)\left[\left(1-\varphi_{2}\right)^{2}-\varphi_{1}^{2}\right]}{1-\varphi_{2}} \frac{\sigma_{c}^{2}}{2 \pi},
\end{aligned}
$$

where $\sigma_{c}^{2}$ is the variance of $\left\{c_{t}\right\} . \omega$ is the angular frequency, measured in radians. The pseudo spectrum of $y_{t}$ can be represented as

$$
\begin{aligned}
g_{y}(\omega) & =[2(1-\cos \omega)]^{-n} \sigma_{\eta}^{2} / 2 \pi+g_{c}(\omega) \\
& =\left(\sigma_{\eta}^{2} / 2 \pi\right)\left\{[2(1-\cos \omega)]^{-n}+\lambda^{*} \tilde{g}_{c}(\omega)\right\},
\end{aligned}
$$

where $\lambda^{*}=\sigma_{c}^{2} / \sigma_{\eta}^{2}$ is the true inverse signal-to-noise ratio and $\tilde{g}_{c}(\omega)$ is defined as $\tilde{g}_{c}(\omega)=$ $2 \pi g_{c}(\omega) / \sigma_{c}^{2}$. 
The pseudo-spectrum $g_{y}(\omega)$ is infinite at $\omega=0$ and can be derived along the arguments presented in Harvey (1989, section 2.4) or Kaiser and Maravall (2001, section 2.5). The key element concerning the trend part is that $\left\{\eta_{t}\right\}$ has a flat spectrum with value $\sigma_{\eta}^{2} / 2 \pi$ and the filter $(1-L)^{-n}$ has the power transfer function $\left[\left(1-e^{-\omega i}\right)\left(1-e^{\omega i}\right)\right]^{-n}=$ $[2(1-\cos \omega)]^{-n}$, where $i=\sqrt{-1}$ is the imaginary unit.

\subsection{The optimal Wiener-Kolmogorov filter}

We consider the Wiener-Kolmogorov filter as the optimal reference filter for the extraction of the trend component (and symmetrically for the extraction of the cycle). It minimizes the mean square error of the estimated component

$$
\operatorname{MSE}_{\hat{\mu}}=E\left(\hat{\mu}_{t}-\mu_{t}\right)^{2},
$$

where $\hat{\mu}_{t}$ is the estimator for the unknown $\mu_{t}$.

It is easy to show that the optimal estimator is given by the conditional expectation, $\hat{\mu}_{t}=E\left(\mu_{t} \mid y\right)$, where $y$ is the vector of all observations. Assuming that all shocks are IID we can express the filter formula as the linear function

$$
\hat{\mu}_{t}=\sum_{j=-\infty}^{\infty} m_{j} y_{t-j},
$$

where the weight $m_{j}$ is given by the coefficient of $L^{j}$ in the polynomial

$$
M(L)=\left(\sigma_{\eta}^{2} /\left|(1-L)^{n}\right|^{2}\right) /\left(\sigma_{\eta}^{2} /\left|\left(1-L^{n}\right)\right|^{2}+\sigma_{\epsilon}^{2} /|\Phi(L)|^{2}\right) .
$$

Here we follow the convention to denote $A(L) A\left(L^{-1}\right)$ as $|A(L)|^{2}$. The formula is an application of the general framework developed by Whittle (1983) and Bell (1984) and described by Harvey (1989) and Kaiser and Maravall (2001). The numerator is the autocovariance generating function of $\left\{\mu_{t}\right\}$, the denominator the autocovariance generating function of $\left\{y_{t}\right\}$.

The power transfer function of the low-pass filter $M(L)$ is obtained by replacing the lag operator $L$ by $e^{-i \omega}$ in $|M(L)|^{2}=M(L) M(-L)$. The gain function $|M(\omega)|=\sqrt{|M(\omega)|^{2}}$ is then given by

$$
|M(\omega)|=\sigma_{\eta}^{2}[2(1-\cos \omega)]^{-n} /\left\{\sigma_{\eta}^{2}[2(1-\cos \omega)]^{-n}+\tilde{g}_{c}(\omega) \sigma_{c}^{2}\right\},
$$

where $\tilde{g}_{c}(\omega)=g_{c}(\omega) \cdot 2 \pi / \sigma_{c}^{2}$ (already defined after equation (5)).

Using the definition $\lambda^{*}=\sigma_{c}^{2} / \sigma_{\eta}^{2}$ (the true inverse signal-to-noise ratio), we can write

$$
|M(\omega)|=1 /\left[1+\lambda^{*}[2(1-\cos \omega)]^{n} \tilde{g}_{c}(\omega)\right] .
$$

Applying the same procedure to the cyclical component, we get

$$
\hat{c}_{t}=(1-M(L)) y_{t}=\sum_{j} h_{j} y_{t-j}=H(L) y_{t} .
$$


The gain function of the high-pass filter $H(L)=1-M(L)$ is given by

$$
|H(\omega)|=1-|M(\omega)| .
$$

The properties of a filter are often assessed by exploring its gain function. However, as Kaiser and Maravall (2001) note, "this function only tells part of the story". It is much more useful to consider the spectrum of a generated component. The spectrum is derived as the product of the squared gain (the power transfer function) and the spectrum of the observed time series (see, e.g., Harvey 1993).

We can derive the spectra of $\hat{\mu}$ and $\hat{c}$ as

$$
g_{\hat{\mu}}(\omega)=|M(\omega)|^{2} g_{y}(\omega)
$$

and

$$
g_{\hat{c}}(\omega)=|H(\omega)|^{2} g_{y}(\omega) .
$$

The spectrum of $(1-L)^{d} \hat{\mu}_{t}$ is given by

$$
g_{\Delta d \hat{\mu}}=[2(1-\cos \omega)]^{d} g_{\hat{\mu}}(\omega),
$$

where $d$ is a positive integer.

\subsection{The Hodrick-Prescott filter}

The application of the Wiener-Kolmogorov filter requires the knowledge of the covariance generating function which depends on the parameters of the true data generating process. As already mentioned in the introduction, many applied researchers avoid the cost of the specification and estimation of an appropriate time series model and use an ad-hoc filter like the Hodrick-Prescott (HP) filter. In the following we summarize the most important properties of this filter. Suppose a doubly infinite series, the cycle is estimated by the high-pass filter (King/Rebelo 1993)

$$
\tilde{c}_{t}=\tilde{H}(L) y_{t},
$$

where

$$
\tilde{H}(L)=\frac{\lambda(1-L)^{2}\left(1-L^{-1}\right)^{2}}{1+\lambda(1-L)^{2}\left(1-L^{-1}\right)^{2}}=\frac{\lambda L^{-2}(1-L)^{4}}{1+\lambda L^{-2}(1-L)^{4}} .
$$

$\lambda$ denotes no longer the true inverse signal-to-noise ratio, but is a prespecified smoothing parameter. The weight function $\tilde{H}(L)$ is equal to the weight function $H(L)$ of the Wiener-Kolmogorov filter when we assume that the cycle is white noise $(\Phi(L)=1), n=$ 2 and $\lambda=\sigma_{\epsilon}^{2} / \sigma_{\eta}^{2}$.

When we replace $L$ by $e^{-i \omega}$ we get the frequency response function $\tilde{H}(\omega)$ of the HP filter. The spectrum of $\tilde{c}_{t}$ is then given by

$$
g_{\tilde{c}}(\omega)=|\tilde{H}(\omega)|^{2} g_{y}(\omega),
$$


where the transfer function $|\tilde{H}(\omega)|^{2}$ is given by $|\tilde{H}(\omega)|^{2}=\tilde{H}(\omega) \tilde{H}(-\omega)$ and $g_{y}(\omega)$ is the pseudo-spectrum of $\left\{y_{t}\right\}$.

$|\tilde{H}(\omega)|^{2}$ can be expressed as

$$
|\tilde{H}(\omega)|^{2}=\left(\frac{4 \lambda(1-\cos \omega)^{2}}{1+4 \lambda(1-\cos \omega)^{2}}\right)^{2} .
$$

The trend is estimated by the low-pass filter

$$
\tilde{\mu}_{t}=\tilde{M}(L) y_{t}=(1-\tilde{H}(L)) y_{t}=\left(1+\lambda(1-L)^{2}\left(1-L^{-1}\right)^{2}\right)^{-1} y_{t} .
$$

The pseudo spectrum of $\tilde{\mu}_{t}$ is given by

$$
g_{\tilde{\mu}}(\omega)=|\tilde{M}(\omega)|^{2} g_{y}(\omega) .
$$

$|\tilde{M}(\omega)|^{2}$ can be expressed as

$$
|\tilde{M}(\omega)|^{2}=\left(1+4 \lambda(1-\cos \omega)^{2}\right)^{-2} .
$$

We can also derive the spectrum of $(1-L)^{d} \tilde{\mu}_{t}$ (where $d$ is a positive integer) as

$$
g_{\Delta d \tilde{\mu}}(\omega)=[2(1-\cos \omega)]^{d} g_{\tilde{\mu}}(\omega) .
$$

As already mentioned the choice of 1600 for the smoothing parameter $\lambda$ seems to be the "industry standard" for quarterly data (for example, this is the default value in EVIEWS). The HP filter is the optimal filter if the trend follows an integrated random walk, the cycle is white noise (and not correlated with the trend shocks) and $\lambda$ is set to the inverse signalto-noise ratio $\sigma_{c}^{2} / \sigma_{\eta}^{2}$ (Kaiser/Maravall 2001). Even if the value of 1600 for $\lambda$ were optimal for US GDP it may be not optimal for GDP of other countries or for other time series like investment (Harvey/Trimbur 2008). The possibly more important and interesting question is whether it is optimal to set $\lambda$ equal to the inverse signal-to-noise ratio in cases when the cycle is not white noise. We will deal with this problem in the next section.

\section{The optimal value for the HP smoothing parameter}

An appropriate choice of the smoothing parameter is crucial for the quality of the HP filter. One approach is based on considerations in the frequency domain (e.g., Pedersen 2001). In this approach an "ideal" filter for the trend is defined as a low-pass filter for which the gain is one for frequencies below a pre-specified cut-off frequency and zero for higher frequencies. The value for the smoothing parameter $\lambda$ is then calculated by minimizing the squared difference between the gain functions of the ideal and of the HP filter, respectively. A variant of this approach is the specification of a cut-off frequency $\omega_{0}$ for which the gain of the HP filter should be 0.5 . $\lambda$ is then given by $\lambda=\left[2 \sin \left(\omega_{0} / 2\right)\right]^{-4}$ (Gomez 2001).

Proietti (2005) determines the "optimal" values for parameters of misspecified models used for signal extraction (a special case is the HP filter) by minimizing the variance of 
multistep forecast errors. He demonstrated for several examples that misspecified models (e.g., the local linear trend model in case the true data generating process contains cyclical elements) may be able to produce reasonable and relatively efficient estimates of unobserved components as trend or cycle of economic time series.

In this paper we follow the lines of Harvey and Trimbur (2008) and match the gain functions of the optimal Wiener-Kolmogorov filter and of the HP filter for different values of $\lambda$. The basic procedure is explained in section 3.1, in section 3.2 the results for several models are presented.

\subsection{The general procedure}

In this section we derive the optimal value for the HP smoothing parameter $\lambda$ in cases where the cyclical component is not white noise but rather follows a stationary autocorrelated process. The basic principle of the derivation is based on the idea that $\lambda$ should be chosen in such a way that the distance between the gain function $|M(\omega)|$ of the optimal Wiener-Kolmogorov filter (the reference filter) and the gain function $|\tilde{M}(\omega)|$ of the $\operatorname{HP}(\lambda)$ filter is as small as possible. If the two gain functions are identical, the two filters have the same filter weights and produce the same output (trend and cycle components). If they are different, the properties of the HP-generated trend and cycle components are not optimal.

The best measure for the distance between two gain functions is the integral of the squared difference between the two functions over all frequencies between 0 and $\pi$. However, the minimization of this measure is a very complicated task. Therefore, we follow a simpler approach and proceed in the following way: Firstly, we specify an AR process for the cycle $\left\{c_{t}\right\}$, derive the spectrum $g_{c}(\omega)$ and use equation (8a) for calculating the gain function $|M(\omega)|$ of the optimal Wiener-Kolmogorov filter. Secondly, from $|M(\omega)|$ we determine numerically the frequency $\omega_{0}$, where the gain has the value $0.5:\left|M\left(\omega_{0}\right)\right|=0.5$. Third, we use the relation (Gomez 2001) $\lambda_{H P}=\left[2 \sin \left(\omega_{0} / 2\right)\right]^{-4}$ for calculating that value of $\lambda$ for which the gain of the HP filter has also a value of 0.5 at frequency $\omega_{0}$ : At frequency $\omega_{0}$, the gain functions of the optimal Wiener-Kolmogorov and of the HP filter intersect. As Harvey and Trimbur (2008) note, this criterion is a satisfactory approximation to minimizing the distance between the two gain functions.

\subsection{Numerical calculations for infinite time series}

In the following we use different specifications of the trend and cycle components for calculating the "optimal" $\lambda_{H P}^{o p t}$ with the procedure outlined in section 3.1. The trend component is alternatively specified as integrated of order one (RW(1)) or of order two (RW(2)). For the cycle component we specify stationary $\operatorname{AR}(1)$ and $\operatorname{AR}(2)$ models. In this section we assume the availability of an infinite time series.

\subsubsection{Trend RW(2) and cycle AR(1)}

The model is given by (see section 2.1)

$$
\begin{aligned}
(1-L)^{2} \mu_{t} & =\eta_{t} \\
c_{t} & =\varphi_{1} c_{t-1}+\epsilon_{t} .
\end{aligned}
$$


Table 1 Optimal $\lambda_{H P}^{\text {opt }}$ for different values of $\varphi_{1}$ and $\sigma_{c}^{2} / \sigma_{\eta}^{2}$ (Trend: RW(2); Cycle: AR(1))

\begin{tabular}{cc|ccc}
\hline & & \multicolumn{3}{|c}{$\sigma_{c}^{2} / \sigma_{\eta}^{2}$} \\
& & 800 & 1600 & 6400 \\
\hline \multirow{4}{*}{$\varphi_{1}$} & 0.0 & $800(1.0)$ & $1600(1.0)$ & $6400(1.0)$ \\
& 0.1 & $975(1.2)$ & $1950(1.2)$ & $7811(1.2)$ \\
& 0.3 & $1462(1.8)$ & $2938(1.8)$ & $11821(1.8)$ \\
& 0.5 & $2304(2.9)$ & $4664(2.9)$ & $18926(3.0)$ \\
& 0.7 & $4039(5.0)$ & $8356(5.2)$ & $34820(5.4)$ \\
& 0.9 & $7439(9.3)$ & $18248(11.4)$ & $94043(14.7)$ \\
\hline
\end{tabular}

Note: The numbers in parentheses denote the ratio $\lambda_{H P}^{o p t} /\left(\sigma_{c}^{2} / \sigma_{\eta}^{2}\right)$

$\eta_{t}$ and $\epsilon_{t}$ are mutually uncorrelated white noise processes. The calculations are carried out for different values of $\varphi_{1}$ and the inverse signal-to-noise ratio $\left(\sigma_{c}^{2} / \sigma_{\eta}^{2}\right)$. Table 1 shows the "optimal" values for the HP smoothing parameter.

Except in cases with rather high values of the autoregressive parameter $\varphi_{1}$, the ratio $\lambda_{H P}^{o p t} /\left(\sigma_{c}^{2} / \sigma_{\eta}^{2}\right)$ does not depend much on the true inverse signal-to-noise ratio $\left(\sigma_{c}^{2} / \sigma_{\eta}^{2}\right)$. However, the optimal $\lambda_{H P}^{o p t}$ increases strongly with the autoregressive parameter $\varphi_{1}$. For $\varphi_{1}=0.5$, a relatively modest degree of autocorrelation, the optimal $\lambda_{H P}^{o p t}$ is about three times higher than the true inverse signal-to-noise ratio. For $\varphi_{1}=0.9, \lambda_{H P}^{o p t}$ is about ten times higher than $\left(\sigma_{c}^{2} / \sigma_{\eta}^{2}\right)$. This implies that the standard value of $\lambda_{H P}=1600$ is in most cases much too low, even when the assumption of Hodrick/Prescott concerning the inverse signal-to-noise ratio $\left(\sigma_{c}^{2} / \sigma_{\eta}^{2}=1600\right)$ is valid.

Figure 1 shows the filter weights of the optimal Wiener-Kolmogorov (WK) filter (continuous line) for the $\operatorname{AR}(1)$ model with $\varphi_{1}=0.7$ and the true inverse signal-to-noise ratio $\left(\sigma_{c}^{2} / \sigma_{\eta}^{2}\right)=1600$ (equation (7)), of the $\mathrm{HP}(1600)$ filter (dashed line) and of the $\operatorname{HP}(8356)$ filter (dotted line) (equation (13)). The $\mathrm{HP}(8356)$ filter is the "optimal" HP filter (in the sense explained above) for the model. The weights for the WK and the $\mathrm{HP}(8356)$ filter are almost identical. Only for the central observation and the first lag and lead the weights for the $\mathrm{HP}(8356)$ filter are slightly lower than the weights for the WK filter. The weights for the standard $\mathrm{HP}(1600)$ filter, however, are far away from the optimal weights.

The pattern of the filter weights carries over to the gain functions of the filters (equations (8a), (15)). Figure 2 shows the gain for the three low-pass filters. The gains of the WK and the $\mathrm{HP}(8356)$ filter, respectively, are contiguous and the distance between the gain functions is low, whereas the gain of the $\mathrm{HP}(1600)$ filter is moved to the right (to higher frequencies). Especially for frequencies between 0.1 and 0.3 (this corresponds to periods of about 60 and 20 quarters) the gain of the $\mathrm{HP}(1600)$ filter is much higher than the gain of the optimal filter. Consequently, the trend extracting $\mathrm{HP}(1600)$ filter is too responsive to fluctuations which are commonly counted as business cycles. Similar results are obtained when we use different values for the autoregressive parameter and the true inverse signalto-noise ratio. In all cases the message remains the same: The best approximation of the optimal Wiener-Kolmogorov filter is achieved by choosing a $\lambda$ much higher than the inverse signal-to-noise ratio $\sigma_{c}^{2} / \sigma_{\eta}^{2}$. 




Figure 1 Filter weights for WK, HP(1600) and HP(8356) filters (Trend: RW(2); Cycle: $A R(1)$; $\left.\varphi_{1}=0.7\right)$

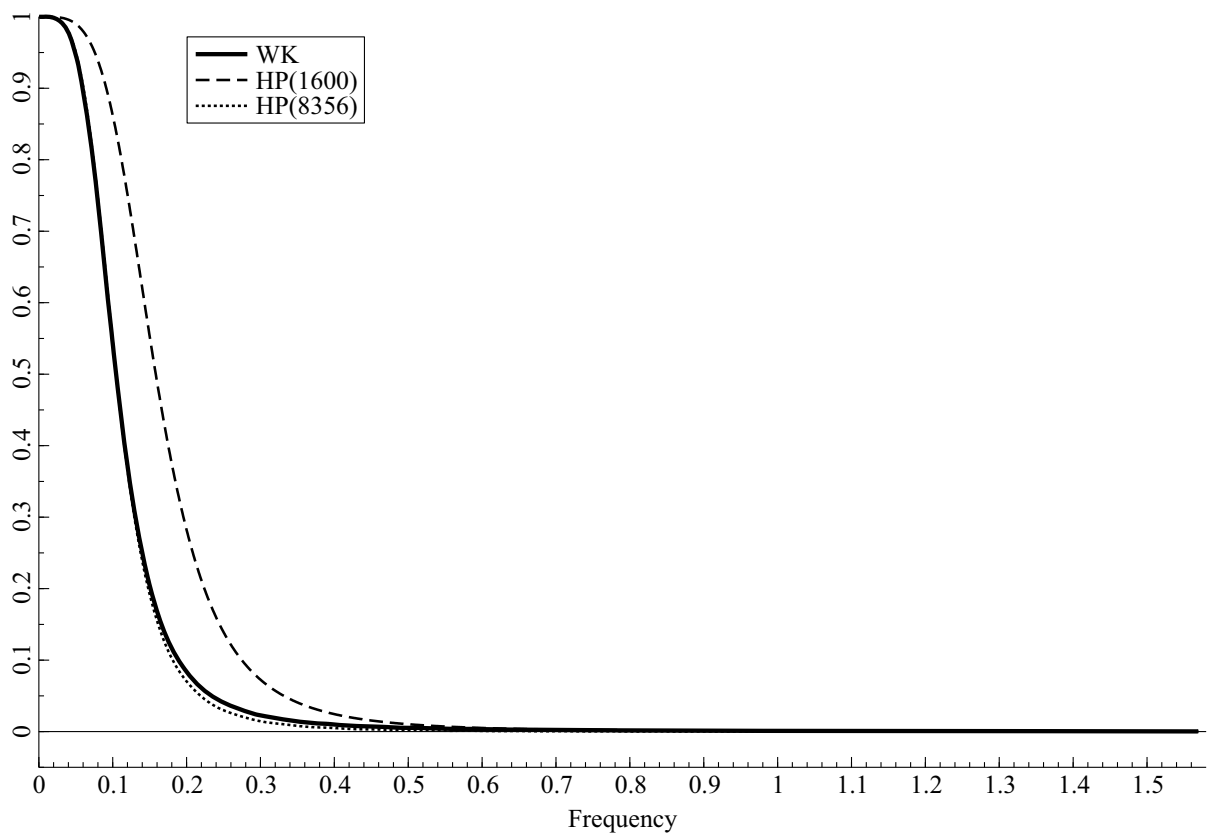

Figure 2 Gain functions of WK, HP(1600) and HP(8356) filters (Trend: RW(2), Cycle: $A R(1)$, $\left.\varphi_{1}=0.7\right)$ 

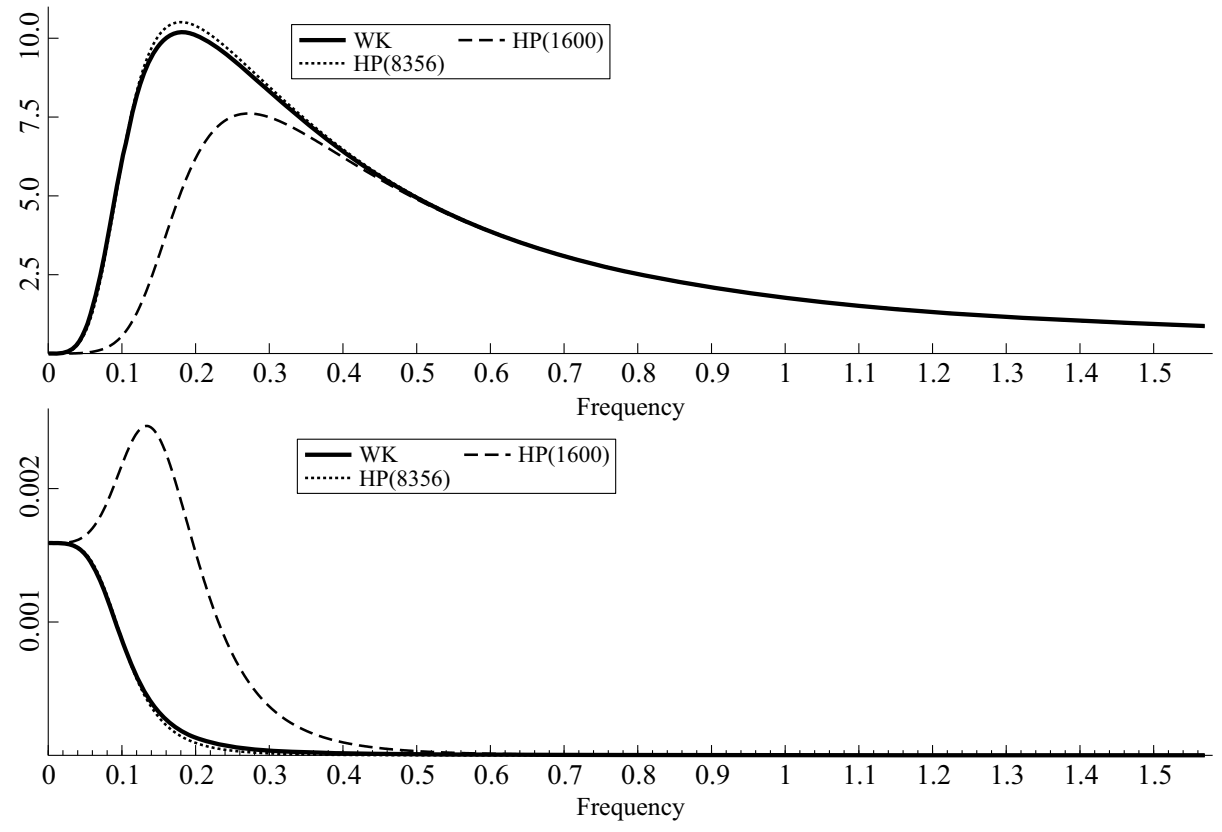

Figure 3 Spectra for the generated cycles (upper part) and for the second differences of generated trends (lower part) (Trend: RW(2); Cycle: $A R(1), \varphi_{1}=0.7$ )

In Figure 3 we evaluate the implied spectra of the generated cycle $\hat{c}$ and of the second differences of the generated trend $\hat{\mu}$ (equations (10), (11), (12) and (16)). The upper part shows the spectra of the generated cycles. The spectrum of the cycle generated with the $\mathrm{HP}(1600)$ filter is again markedly different from that of the cycles generated with optimal filters. As the variance of a stationary time series is proportional to the integral of the spectrum, it is clear that the variance of the cycle generated by $\operatorname{HP}(1600)$ is much lower than the variance of the cycles generated by the $\mathrm{WK}$ and the $\mathrm{HP}(8536)$ filters. In addition, the peak of the spectrum of the cycle generated by $\mathrm{HP}(1600)$ is at a higher frequency. Consequently, the $\mathrm{HP}(1600)$ produces shorter and smaller cycles than the optimal filters generate.

An analogous pattern applies for the spectra of the second differences of the generated trends (lower part of Figure 3). Most important is that the $\mathrm{HP}(1600)$ filter produces a spectrum of $(1-L)^{2} \hat{\mu}_{t}$ with a pronounced peak in the region of business cycle frequencies: A value for $\lambda$ "too low" produces cycles in the second differences of the estimated trend. We will argue below that this feature can be used in practical applications for determining a reasonable value for the smoothing parameter.

\subsubsection{Trend $\mathrm{RW}(2)$ and cycle $\mathrm{AR}(2)$}

The model is now given by

$$
\begin{aligned}
(1-L)^{2} \mu_{t} & =\eta_{t} \\
c_{t} & =\varphi_{1} c_{t-1}+\varphi_{2} c_{t-2}+\epsilon_{t} .
\end{aligned}
$$


There are many combinations of $\varphi_{1}$ and $\varphi_{2}$ compatible with the stationarity assumption. In the following we restrict the analysis to four combinations with complex roots in the AR polynomial: 1.) $\varphi_{1}=1.109, \varphi_{2}=-0.36$; 2.) $\varphi_{1}=1.663, \varphi_{2}=-0.81$; 3.) $\varphi_{1}=1.177, \varphi_{2}=-0.36$; 4.) $\varphi_{1}=1.765, \varphi_{2}=-0.81$. The parameters are chosen accordingly to the AR part of a structural time series model (Harvey 1993, section 6.5). We set $\varphi_{1}=2 \rho \cos \omega_{c}$ and $\varphi_{2}=-\rho^{2}$, where $|\rho|<1$ is a damping factor and $\omega_{c}$ is the frequency of a cyclical function. The roots of the AR polynomial are a pair of complex conjugates. Model 1 has a damping factor $\rho$ of 0.6 and a frequency $\omega_{c}$ of 0.393 (16 quarters), model 2 a damping factor of 0.9 and a frequency of 0.393 , model 3 a damping factor of 0.6 and a frequency of 0.196 (32 quarters) and model 4 a damping factor of 0.9 and a frequency of 0.196 . We have two models with a short and two models with a long cycle, combined with two different damping factors, 0.6 and 0.9 , respectively.

Table 2 shows the optimal values for the HP smoothing parameter for the different models and three different values of the true inverse signal-to-noise ratio (800, 1600 and 6400). In all cases the optimal value for $\lambda$ is much higher than $\sigma_{c}^{2} / \sigma_{\eta}^{2}$. For parameter combination 1.) it is about 4.5 times higher, for combination 2.) about 3 times higher, for combination 3.) about 6 times higher and for combination 4.) about 10 times higher. For instance, if we assume a pronounced cycle with a period of 8 years and a true inverse signal-to-noise ratio of 1600 , the optimal value for the HP smoothing parameter is 15887 !

Table 2 Optimal $\lambda_{H P}^{\text {opt }}$ for different values of $\varphi_{1}, \varphi_{2}$ and $\sigma_{c}^{2} / \sigma_{\eta}^{2}$ (Trend: RW(2); Cycle: AR(2))

\begin{tabular}{cc|ccc}
\hline & & \multicolumn{3}{|c}{$\sigma_{c}^{2} / \sigma_{\eta}^{2}$} \\
& & 800 & 1600 & 6400 \\
\hline \multirow{4}{*}{$\varphi_{1}, \varphi_{2}$} & $1.109,-0.36$ & $3627(4.5)$ & $7297(4.6)$ & $29385(4.6)$ \\
& $1.663,-0.81$ & $2476(3.1)$ & $4657(2.9)$ & $17233(2.7)$ \\
& $1.177,-0.36$ & $4871(6.1)$ & $9944(6.2)$ & $40753(6.4)$ \\
& $1.765,-0.81$ & $8379(10.5)$ & $15887(9.9)$ & $58905(9.2)$ \\
\hline
\end{tabular}

Note: The numbers in parentheses denote the ratio $\lambda_{H P}^{o p t} /\left(\sigma_{c}^{2} / \sigma_{\eta}^{2}\right)$

Figure 4 shows the filter weights of the optimal Wiener-Kolmogorov (WK) filter (continuous line) for the $\operatorname{AR}(2)$ model with $\varphi_{1}=1.765, \varphi_{2}=-0.81$ and $\sigma_{c}^{2} / \sigma_{\eta}^{2}=1600$, of the standard $\mathrm{HP}(1600)$ filter (dashed line) and of the $\mathrm{HP}(15887)$ filter (dotted line). Contrary to the $\mathrm{AR}(1)$ case, the weighting pattern of the WK filter can not be fully replicated by a HP filter with a suitably chosen $\lambda$. The reason is that the weight function of the HP filter is always relatively smooth, whereas the weight function of the WK filter has in the model under consideration a spike for the central observation (this spike occurs only in cases of a high value for the damping factor $\rho$ ). However, the difference between the weights of the WK and of the $\mathrm{HP}(15887)$ filter is very small for lags and leads higher than four. In contrast, the shape of the weights of the $\mathrm{HP}(1600)$ filter is very different (the near coincidence with the weight of the WK filter for the central observation is accidental).

Figure 5 shows the gain for the three low-pass filters. For frequencies lower than about 0.25 (a period of about 6 years) the gain function of the WK and the HP(15887) filters are almost identical. For higher frequencies, the gain of the $\mathrm{HP}(15887)$ filter converges to zero, whereas the gain of the WK filter has small positive values. Similarly to the AR(1) model, the gain function of the traditional $\mathrm{HP}(1600)$ filter is very different from the gain 


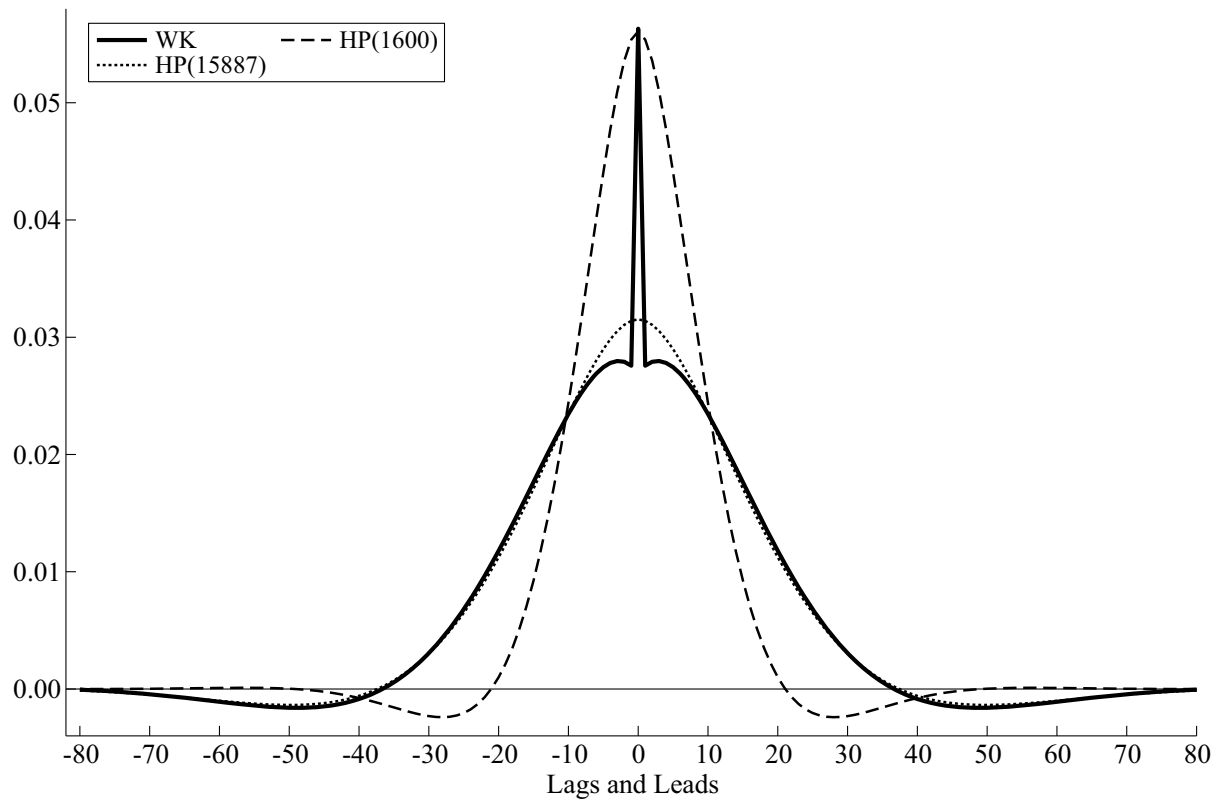

Figure 4 Filter weights for the WK, HP(1600) and HP(15887) filters. (Trend: RW(2); Cycle: $\left.\mathrm{AR}(2), \varphi_{1}=1.765, \varphi_{2}=-0.81\right)$

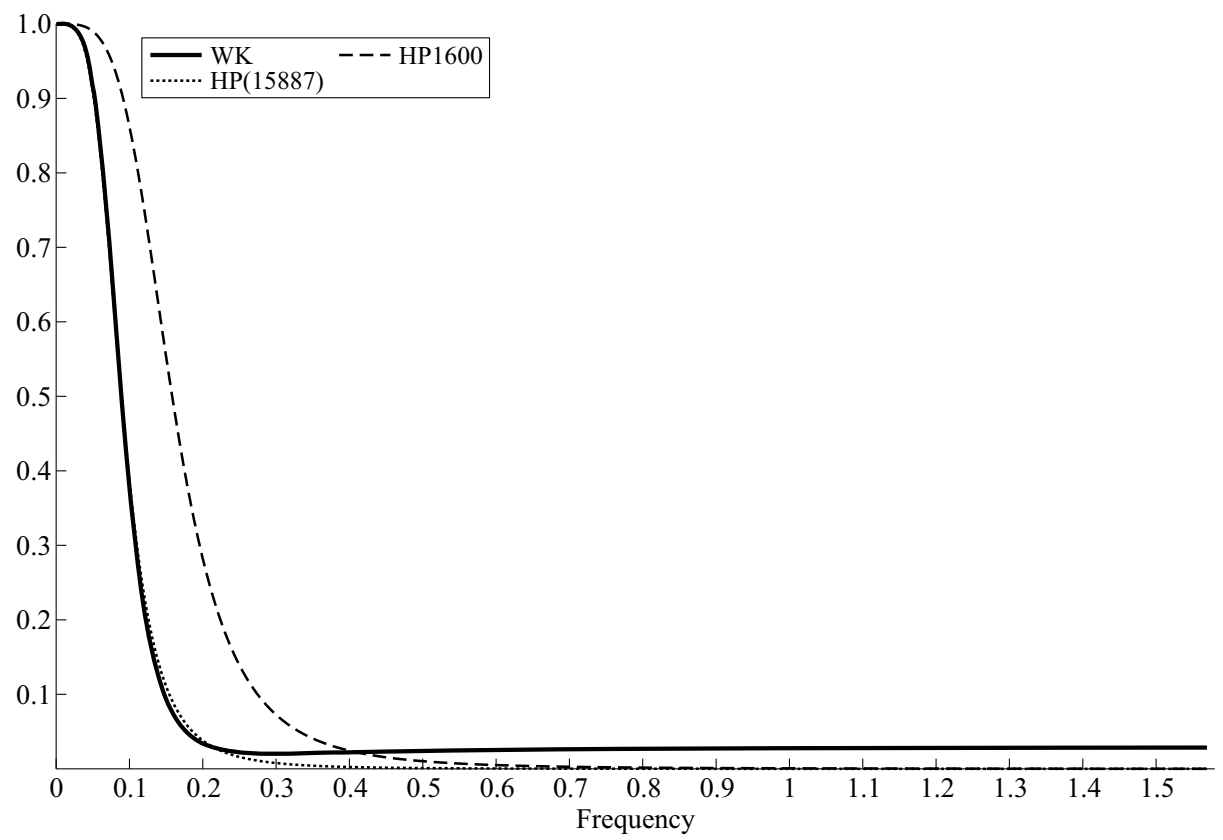

Figure 5 Gain functions of WK, HP(1600) and HP(15887) filters (Trend: RW(2); Cycle: AR(2), $\varphi_{1}=1.765, \varphi_{2}=-0.81$ ) 


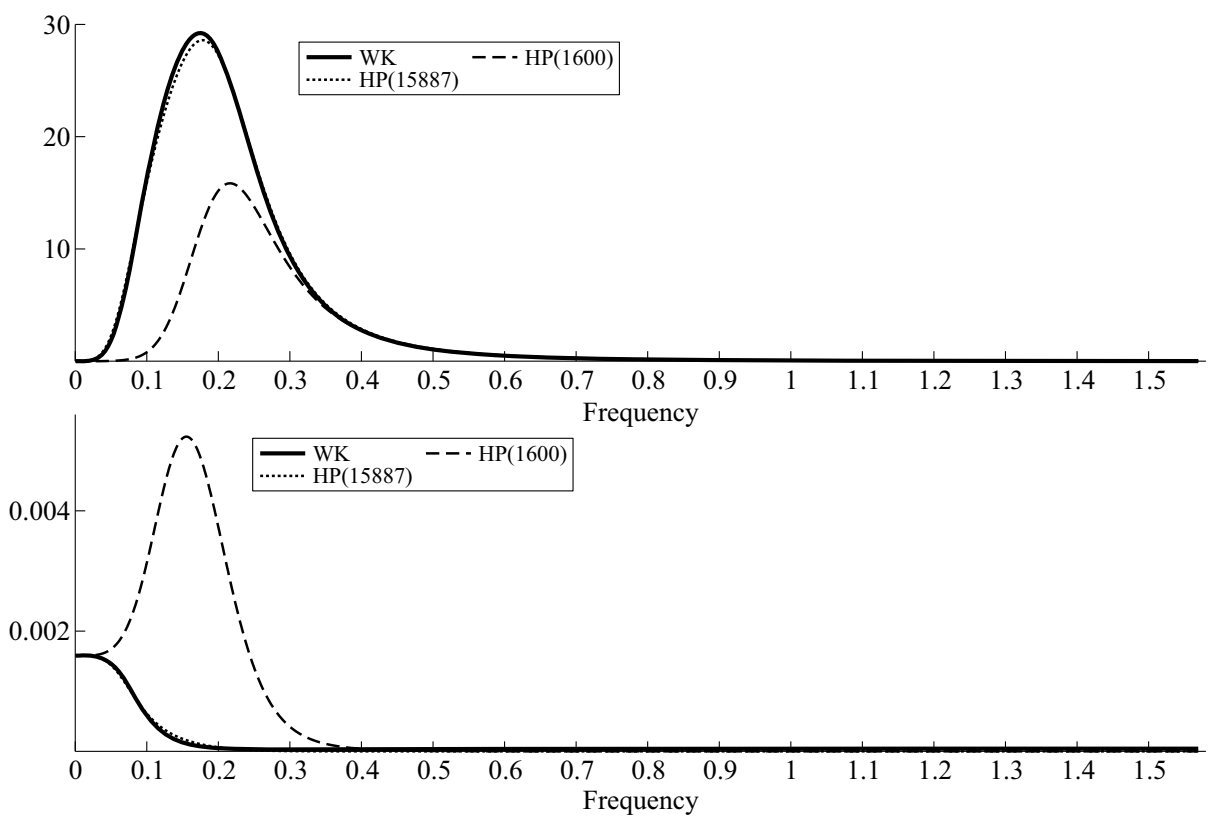

Figure 6 Spectra for the generated cycles (upper part) and for the second differences of generated trends (lower part). (Trend: $\operatorname{RW}(2)$; Cycle: $\operatorname{AR}(2), \varphi_{1}=1.765, \varphi_{2}=-0.81$ )

of the WK filter. It is moved to higher frequencies. Again, the generated trend is too responsive to fluctuations with business cycle properties.

Figure 6 shows the spectra of the generated cycles (upper part) and of the second differences of the generated trend (lower part). Again, the spectra are very similar for the components generated by the WK filter and the "optimal" $\mathrm{HP}(15887)$ filter, whereas the spectra generated by using the traditional $\mathrm{HP}(1600)$ filter are very different. The cycle is clearly underestimated by $\mathrm{HP}(1600)$ and the spectrum of the second differences of the estimated trend shows a very pronounced peak in the region of business cycle frequencies. The trend has undesirable cyclical features.

\subsubsection{Trend RW(1)}

In case the trend is a random walk (integrated of order one) the HP filter delivers relatively poor results. The conclusion from exercises shown in an Appendix (available in the data archive of the journal, www.jbnst.de/en) is that the HP filter does not work satisfactorily when the trend follows a random walk. In this case exponential smoothing may be a much better choice.

\subsection{A simulation study}

The results in the previous sections are derived under the assumption of a double infinite time series. In finite time series we have at the start and the end of the sample asymmet- 
ric filters. In order to check the ability of the HP filter with a relatively high smoothing parameter to replicate the main properties of the WK filter for autocorrelated cycle processes we carry out a simulation study for a finite time series with 160 observations. The model is given by

$$
\begin{aligned}
y_{t} & =\mu_{t}+c_{t} \\
(1-L)^{2} \mu_{t} & =\eta_{t} \\
c_{t} & =\varphi_{1} c_{t-1}+\varphi_{2} c_{t-2}+\epsilon_{t}
\end{aligned}
$$

$\eta_{t}$ and $\epsilon_{t}$ are mutually uncorrelated white noise processes. The inverse signal-to-noise ratio $\sigma_{c}^{2} / \sigma_{\eta}^{2}$ is set to the three alternative values 800,1600 and 6400 .

We generate series for $\left\{\mu_{t}\right\},\left\{c_{t}\right\}$ and $\left\{y_{t}\right\}, t=1, \ldots, 160$ and filter the "observed" time series $\left\{y_{t}\right\}$ with the optimal WK filter and the HP filter, using for the latter different values of the smoothing parameter $\lambda$.

The trend values $\hat{\mu}$ estimated by the WK filter are generated by using the matrix formula (McElroy 2008):

$$
\hat{\mu}=\left(C_{c}^{-1}+\lambda^{*} D^{\prime} D\right)^{-1} C_{c}^{-1} y
$$

$C_{c}$ is the $T \times T$ correlation matrix of $\left\{c_{t}\right\}, \lambda^{*}$ is the true inverse signal-to-noise ratio $\sigma_{c}^{2} / \sigma_{\eta}^{2}$ and $D$ is the $(T-2) \times T$ differencing matrix, given by

$$
D=\left(\begin{array}{cccccccc}
1 & -2 & 1 & 0 & \cdots & 0 & 0 & 0 \\
0 & 1 & -2 & 1 & \cdots & 0 & 0 & 0 \\
\vdots & \vdots & \vdots & \vdots & & & & \\
0 & 0 & 0 & 0 & \cdots & 1 & -2 & 1
\end{array}\right)
$$

For estimating $\mu$ with the HP filter we set $C_{c}=I$ and $\lambda^{*}$ to a prespecified value of the smoothing parameter $\lambda$.

The simulation study consists of 1000 replications of the described procedure. For each replication we calculated the mean square error $M S E=\left(\sum_{t}\left(\mu_{t}-\hat{\mu}_{t}\right)^{2}\right) / T$ for the different filters.

Following Harvey and Delle Monache (2009), we assess the efficiency of a filter by the ratio $M S E_{W K} / M S E_{H P}$, where $M S E_{W K}$ and $M S E_{H P}$ are the mean square error of the Wiener-Kolmogorov filter and the HP filter, respectively.

Table 3 shows the relative efficiency of different HP filters compared to the WK filter for different values of $\varphi_{1}$ for an $\operatorname{AR}(1)$ model of the cycle and for different values of the true inverse signal-to-noise ratio $\sigma_{c}^{2} / \sigma_{\eta}^{2}$.

The entries in Table 3 are obtained in the following way: For each model (characterized by $\varphi_{1}$ and $\sigma_{c}^{2} / \sigma_{\eta}^{2}$ ) we generate 1000 series of 160 observations for trend $\mu$, cycle $c$ and the "observed" time series $y$ (trend + cycle). For each time series we calculate the mean square error MSE $=\left(\sum_{t}\left(\mu_{t}-\hat{\mu}_{t}\right)^{2}\right) / T$ for the WK filter and for 15 HP filters with smoothing parameter $\lambda_{j}=j \sigma_{c}^{2} / \sigma_{\eta}^{2}, j=1,2, \ldots, 15$. An entry in Table 4 shows the mean of 1000 values for $M S E_{W K} / M S E_{H P}$. For each true inverse signal-to-noise ratio (800, 1600 and $6400)$ there are two columns of results. The first column shows the relative efficiency of 
Table 3 Relative efficiency of different HP filters compared to WK filter (Trend: RW(2); Cycle: $\mathrm{AR}(1)$

\begin{tabular}{cc|cccccc}
\hline & & \multicolumn{2}{|c}{800} & \multicolumn{2}{c}{$\sigma_{c}^{2} / \sigma_{\eta}^{2}$} \\
& & \multicolumn{2}{c}{1600} & \multicolumn{2}{c}{6400} \\
& & $\mathrm{HP}(800)$ & $\mathrm{HP}(\mathrm{opt})$ & $\mathrm{HP}(1600)$ & $\mathrm{HP}(\mathrm{opt})$ & $\mathrm{HP}(6400)$ & $\mathrm{HP}(\mathrm{opt})$ \\
\hline & 0.0 & 1.000 & $1.000(1)$ & 1.000 & $1.000(1)$ & 1.000 & $1.000(1)$ \\
$\varphi_{1}$ & 0.1 & 0.996 & $0.996(1)$ & 0.994 & $0.994(1)$ & 0.996 & $0.996(1)$ \\
& 0.3 & 0.964 & $0.996(2)$ & 0.970 & $0.995(2)$ & 0.972 & $0.992(2)$ \\
& 0.5 & 0.912 & $0.990(3)$ & 0.914 & $0.992(3)$ & 0.912 & $0.992(3)$ \\
& 0.7 & 0.829 & $0.984(5)$ & 0.828 & $0.984(5)$ & 0.814 & $0.984(5)$ \\
& 0.9 & 0.796 & $0.964(10)$ & 0.740 & $0.953(12)$ & 0.682 & $0.943(14)$ \\
\hline
\end{tabular}

Note: The numbers in parentheses denote the ratio of the "optimal" HP smoothing parameter to the true inverse signal-to-noise ratio.

the $\operatorname{HP}\left(\sigma_{c}^{2} / \sigma_{\eta}^{2}\right)$ filter, the second the relative efficiency when we choose the "optimal" $\lambda$. The numbers in parentheses denote the ratio of the "optimal" smoothing parameter to $\sigma_{c}^{2} / \sigma_{\eta}^{2}$.

The results indicate that in case the cycle follows an $\mathrm{AR}(1)$ process one can get an impressive efficiency gain by choosing an HP smoothing parameter higher than the true inverse signal-to-noise ratio. Take, for example, a model with $\sigma_{c}^{2} / \sigma_{\eta}^{2}=1600$ and $\varphi_{1}=0.7$. Compared with the WK filter, the $\mathrm{HP}(1600)$ filter has a relative efficiency of 0.83 , whereas the $\mathrm{HP}(8000)$ filter has a relative efficiency of 0.98 .

Table 4 reports the results for four AR(2) processes (see section 3.2.2 for the choice of the parameters). The results confirm the conclusions for the $\operatorname{AR}(1)$ case. By choosing a reasonably high value of the HP smoothing parameter one can achieve a remarkable efficiency gain compared with the HP filter where $\lambda$ is set to the true inverse signal-to-noise ratio.

Table 4 Relative efficiency of different HP filters compared to WK filter (Trend: RW(2); Cycle: $\operatorname{AR}(2))$

\begin{tabular}{rl|llllll}
\hline & \multicolumn{2}{|c}{800} & \multicolumn{2}{c}{$\sigma_{c}^{2} / \sigma_{\eta}^{2}$} & \multicolumn{2}{c}{6400} \\
& & HP(800) & HP(opt) & HP(1600) & HP(opt) & HP(6400) & HP(opt) \\
\hline & $1.109,-0.36$ & 0.835 & $0.980(5)$ & 0.837 & $0.980(5)$ & 0.819 & $0.974(5)$ \\
$\varphi_{1}, \varphi_{2}$ & $1.663,-0.81$ & 0.810 & $0.935(3)$ & 0.828 & $0.949(3)$ & 0.841 & $0.943(3)$ \\
& $1.177,-0.36$ & 0.794 & $0.972(6)$ & 0.779 & $0.968(6)$ & 0.778 & $0.962(6)$ \\
& $1.765,-0.81$ & 0.629 & $0.927(11)$ & 0.626 & $0.912(10)$ & 0.640 & $0.919(10)$ \\
\hline
\end{tabular}

Note: The numbers in parentheses denote the ratio of the "optimal" HP smoothing parameter to the true inverse signal-to-noise ratio.

Compared with the AR(1) model for the cycle the maximal efficiency is now somewhat lower. For instance, for case $4\left(\varphi_{1}=1.765, \varphi_{2}=-0.81\right)$ and a true inverse signal-tonoise ratio of 1600 , the relative efficiency (compared to the WK filter) is $91 \%$. However, 
the $\mathrm{HP}(1600)$ filter has only a relative efficiency of $63 \%$. The reward of using a high smoothing parameter is still high.

The results generated by the simulation study using a finite length of the time series confirm the conclusions of the theoretical analysis for doubly infinite series: When the stationary component of a time series is (positively) autocorrelated, the optimal value for the HP smoothing parameter is several times higher than the inverse signal-to-noise ratio.

\section{Choosing $\lambda$ in practical applications: A new proposal}

It is common knowledge that the HP filter may induce spurious cycles (Cogley/Nason 1995). An often used example is the case of a random walk as the input series (Kaiser/ Maravall 2001). In this case, the HP filter typically produces spurious cycles with periods between 8 and 10 years $(\lambda=1600)$. The main argument in this paper concentrates on the contrary danger that the HP filter dampens true cycles and produces cyclical features of the generated trend. The basic assumption is that the cyclical component is not white noise but follows an autocorrelated process. In this case it is necessary to choose a value for the smoothing parameter $\lambda$ that is much higher than the true or assumed inverse signalto-noise ratio. The problem for the practitioner is that we do not know the parameters of the data-generating process (at least in situations where it is too difficult or too costly to estimate the parameters of structural models).

In this section we propose the following (partial) solution to this problem. We start with the assumption that the generated trend component should not exhibit any cyclical features. Since the trend is not stationary, we concentrate on the first and/or second differences of the trend. We identify a possible cycle in the differences of the generated trend using the spectrum of d-th differences $(1-L)^{d} \tilde{\mu}_{t}, d=1,2$, where $\tilde{\mu}_{t}$ is the HP-generated trend component.

Using the results in section 2.3 , we can write the spectrum $g_{\Delta d \tilde{\mu}}$ of $(1-L)^{d} \tilde{\mu}_{t}$ as

$$
\begin{aligned}
g_{\Delta d \tilde{\mu}} & =[2(1-\cos \omega)]^{d}|\tilde{M}(\omega)|^{2} g_{y}(\omega) \\
& =\left(\sigma_{n}^{2} / 2 \pi\right)\left[1+4 \lambda(1-\cos \omega)^{2}\right]^{-2}\left\{[2(1-\cos \omega)]^{d-n}+[2(1-\cos \omega)]^{d} \lambda^{*} \tilde{g}_{c}(\omega)\right\},
\end{aligned}
$$

where $\lambda$ is the HP smoothing parameter and $\lambda^{*}$ is the true inverse signal-to-noise ratio. If $d<n,(1-L)^{d} \tilde{\mu}_{t}$ is not stationary. Given the parameters of the true data-generating process, the shape of $g_{\Delta d \tilde{\mu}}$ is determined by the smoothing parameter $\lambda$. In the following we concentrate on the case $d=n$. If $\lambda=0, g_{\Delta d \tilde{\mu}}$ is an increasing function of $\omega$, if $\lambda$ is very high, $g_{\Delta d \tilde{\mu}}$ is a decreasing function of $\omega$. For values in between, it is possible that $g_{\Delta d \tilde{\mu}}$ has a peak for $0<\omega<\pi$. If this occurs, we have cycles in the differences of the generated trend.

Figure 7 shows the spectra of $g_{\Delta 2 \tilde{\mu}}$ (second differences of the generated trend) for a model where the trend is an integrated random walk $(n=2)$ and the cycle follows an $\operatorname{AR}(1)$ process with $\varphi_{1}=0.7$ and the true inverse signal-to-noise ratio is 1600 . The thick continuous line shows the spectrum for the $\mathrm{HP}(1600)$ filter. It has a pronounced peak at frequency 0.133 (which corresponds to a period of 47 quarters). The dashed line shows the spectrum for the $\mathrm{HP}(3200)$ filter. We have a peak at frequency 0.091 , which is less pronounced than the peak for the $\mathrm{HP}(1600)$. The spectrum of the $\mathrm{HP}(4800)$ filter (dotted line) has no peak, but is nevertheless shifted to the right compared to the WK filter (thin 


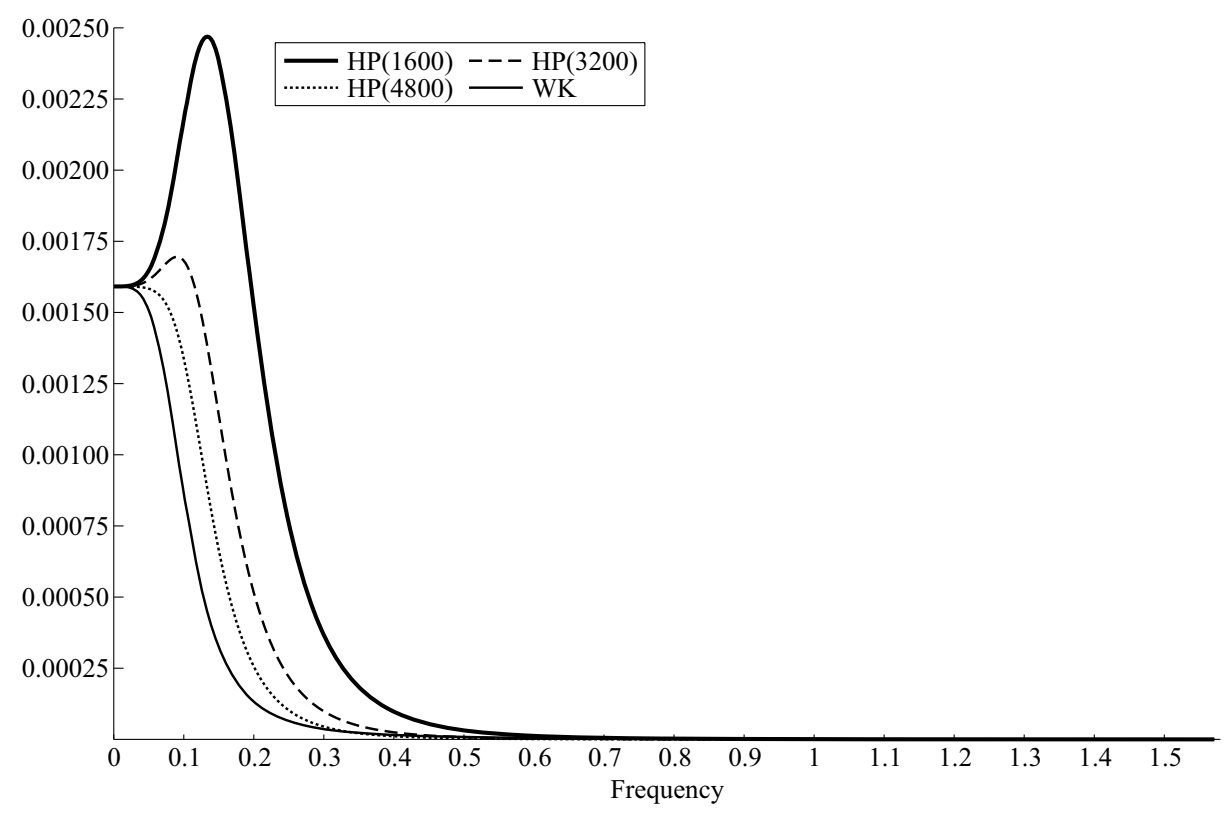

Figure 7 Spectra of second differences of generated trends (HP filter) for different values of $\lambda$ (Trend: RW(2); Cycle: $\operatorname{AR}(1), \varphi_{1}=0.7$ )

continuous line). The trend generated by the $\mathrm{HP}(4800)$ is still to responsive to fluctuations with business cycle frequencies. We know that the "optimal" value for $\lambda$ in this case is 8356 (see section 3.2.1). We conclude that the lowest value for $\lambda$ that does not generate a peak in the spectrum of the second differences of the extracted trend is about half as high as the "optimal" value. This rule of thumb is roughly confirmed by calculations for other $\operatorname{AR}(1)$ or $\operatorname{AR}(2)$ models of the cycle.

\section{An empirical example: US Private Investment}

In this section we study the effects of different values of the HP smoothing parameter on the properties of the estimated trend of US Real Gross Private Domestic Investment (logarithmic values; 1950:1-2011:4; Source: FRED (http://research.stlouisfed.org/fred2/)). We estimate the trend component using the HP filter with values for $\lambda$ of $1600,8000,16000$ and 32000 . Figure 8 shows in the upper part the growth rates for the generated trends, in the lower part changes in the growth rates (second differences). The thick continuous line shows the results for the $\mathrm{HP}(1600)$ filter. Both first and second differences display clearly cycles with a period of about 8 years. Oscillations with this period are counted by many economists as business cycles. If one accepts that business cycles can appear in the growth rates of the trend, it is fine. But the general definition of the trend does not allow for cyclical elements. In this interpretation, the generated "trend" is a mixture of trend and cycle and, consequently, an artifact of the filter. 

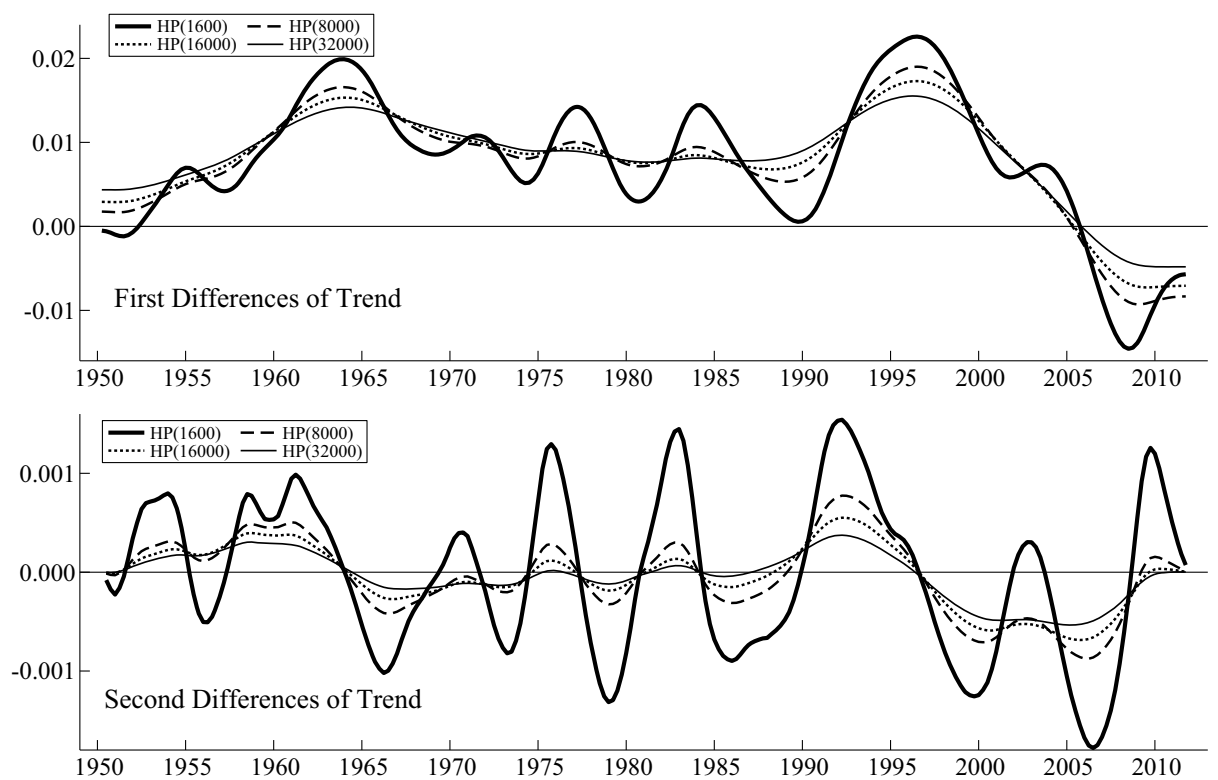

Figure 8 First and second differences of HP generated trends (US Private Investment)

To a lesser degree, the first and second differences of trends generated by the $\mathrm{HP}(8000)$ (dashed line) and $\mathrm{HP}(16000)$ (dotted line) show a similar picture. When we use $\mathrm{HP}(32000)$, the differences display no apparent cyclical element.

From the perspective of the criterion that the trend and its differences should not exhibit any form of a cycle it is clear from the previous discussion that for real investment a smoothing parameter of 1600 is not appropriate. The minimum reasonable values for $\lambda$ is in the region of 20000 to 40000 . Harvey and Trimbur (2008) suggest a value of 32000 (based on a somewhat different line of arguments). Using the result of section 4 one can argue that the "optimal" value may be even higher, say about 60000 .

The choice of the smoothing parameter has far-reaching consequences for the size and the dynamic properties of the HP-generated cycle component. In the example of US Private Investment, the standard deviation of the cycle is 0.077 for $\lambda=1600$ and 0.094 for $\lambda=32000$. And the autocorrelation function decays at a much slower rate for higher $\lambda$ values. For instance, the autocorrelation coefficient at lag 1 (4) is $0.80(0.03)$ for $\lambda=1600$ and $0.87(0.29)$ for $\lambda=32000$. It is left for future research to analyze the implications of different smoothing parameters for other variables (GDP, consumption, employment, etc.) and to explore the consequences for the construction of "stylized facts" of the business cycle.

\section{Summary and conclusions}

When we interpret the Hodrick-Prescott filter as a model-based filter it is the optimal Wiener-Kolmogorov filter (in the sense of minimizing the MSE) if the trend follows an integrated random walk, the cycle is white noise and the smoothing parameter $\lambda$ is set 
to the inverse signal-to-noise ratio. In the traditional trend-cycle decomposition these assumptions are in many cases clearly implausible and the HP filter lacks a sound theoretical foundation.

In this paper we concentrate on the situation where the cycle follows an autocorrelated $\mathrm{AR}(1)$ or $\mathrm{AR}(2)$ process and ask the question whether it is possible to reach a reasonable approximation of the optimal Wiener-Kolmogorov filter by the HP filter with an appropriate chosen value for the smoothing parameter $\lambda$. The analysis is done in the following way: First, we calculate from the gain function of the optimal Wiener-Kolmogorov filter the frequency where the gain is 0.5 . Secondly, we determine the value of $\lambda$ for which the gain function of the HP filter has also the value of 0.5 at the same frequency. In the last step we compare the mean square error of both filters (the WK filter and the HP filter with the "optimized" value of $\lambda$ ). These calculations are carried out for different specifications of the AR-parameters of the cycle, different values of the inverse signal-to-noise ratio and different specifications of the trend component.

The general result is that in case the trend follows an integrated random walk (it is integrated of order two) one gets a good approximation of the weights and the gain function of the optimal Wiener-Kolmogorov filter by choosing a value for the HP smoothing parameter much higher than the inverse signal-to-noise ratio. For example, when the cycle follows an $\operatorname{AR}(1)$ process with a parameter $\varphi_{1}=0.9$, the "optimal" value of $\lambda$ is more than 10 times higher than the true inverse signal-to-noise ratio. The HP filter is an almost optimal filter, provided the smoothing parameter is not too low.

Smoothing parameters "too low" have a twofold distortionary effect. They produce trends with first and/or second differences which exhibit cyclical features. The trend is too responsive to business cycle fluctuations. This implies that the variance and the period of the generated cycle are too low. The relevance of the cyclical component is underestimated.

When the trend is a random walk the approximation is not really satisfactory. It is not possible to replicate the shape of the weight and gain functions of the optimal WienerKolmogorov filter by the HP filter. However, the general result remains that we should select higher values for $\lambda$ than than the inverse signal-to-noise ratio.

These findings are useful, but not really applicable in practice as we do not know the true inverse signal-to-noise ratio. The problem is how to choose an appropriate value for the HP smoothing parameter. The suggestion proposed in this paper is to rely on the properties of the first and/or second differences of the extracted trend. The proposal is based on the assumption that the differences of the extracted trend should not show any cyclical behavior (in the sense that the spectrum of the differences has a peak in the region of business cycle frequencies). It is shown that by choosing a high enough smoothing parameter a peak can always be avoided. In practical applications we could estimate the trend by applying the HP filter with different values for $\lambda$ and search for the lowest among them which does not produce cycles in the differences of the generated trend. Admittedly, this is a preliminary and in some sense vague suggestion. There may be too much room for subjective and discretionary decisions. A possible solution is the analysis in the frequency domain by estimating the spectrum of the first and/or second differences of the generated trend. We can define a range of frequencies of business cycle fluctuations and test for a peak of the empirical spectrum of the trend differences in this interval. If there is a peak, $\lambda$ should be increased until the peak disappears. It is left for 
future research to develop along these lines an efficient procedure which is easy to use also by practitioners.

In the last section the proposed procedure is applied to US private real investment (quarterly data). We find that the lowest value for $\lambda$ that does not produce cycles in the differences of the trend component is about 32000. With some caution, we can conclude that the "optimal" value of the smoothing parameter may be even higher.

The general conclusion of this paper is that the "industry standard" of $\lambda=1600$ for quarterly data may be much too low for many macroeconomic time series (for a discussion of the relationship between smoothing parameters for different frequencies of observations see Ravn/Uhlig 2002). In order to produce a reasonable and reliable trend-cycle decomposition much higher values are necessary. It is left for future research to explore the implications for the "stylized facts" of business cycles. A further important research topic is the analysis of the implications of different values for the smoothing parameter for the estimation at the end of a time series (real time analysis). At the end of a series the HP filter is asymmetric with phase shifts and a different pattern of the the gain function (for an example see Blöchl/Flaig 2014). A comparison between the properties of the optimal Wiener-Kolmogorov filter and the HP filter with different values of $\lambda$ at the end of a sample may be very useful especially for business cycle forecasters.

\section{References}

Bell, W. (1984), Signal Extraction for nonstationary time series. Annals of Statistics 12: 646-664.

Beveridge, S., C.R. Nelson (1981), A New Approach to Decomposition of Economic Time Series into Permanent and Transitory Components with Particular Attention to Measurement of the "Business Cycle". Journal of Monetary Economics 7: 151-174.

Blöchl, A., G. Flaig (2014), The Hodrick-Prescott Filter with a time-varying penalization parameter. An application to the trend estimation of global temperature. CESifo Working Paper No. 4577.

Box, G.E.P., S.C. Hillmer, G.C. Tiao (1978), Analysis and Modeling of Seasonal Time Series. Pp. 309-344 in: A. Zellner (ed.), Seasonal Analysis of Economic Time Series. U.S. Department of Commerce.

Cogley, T., J. Nason (1995), Effects of the Hodrick-Prescott filter on trend and difference stationary time series. Implications for business cycle research. Journal of Economic Dynamics and Control 19: 253-278.

Gomez, V. (1999), Three Equivalent Methods for Filtering Finite Nonstationary Time Series. Journal of Business \& Economic Statistics 17: 109-116.

Harvey, A. (1989), Forecasting, Structural Time Series Models and the Kalman Filter. Cambridge University Press.

Harvey, A. (1993), Time Series Models. MIT Press.

Harvey, A., A. Jaeger (1993), Detrending, Stylized Facts and the Business Cycle. Journal of Applied Econometrics 8: 231-247.

Harvey, A., T. Trimbur (2008), Trend Estimation and the Hodrick-Prescott Filter. Journal of the Japan Statistical Society 38: 41-49.

Harvey, A., D. Delle Monache (2009), Computing the Mean Square Error of Unobserved Components Extracted by Misspecified Time Series Models. Journal of Economic Dynamics \& Control 33. 283-295.

Hodrick, R., E. Prescott (1997), Postwar U.S. Business Cycles. An Empirical Investigation. Journal of Money, Credit, and Banking 29: 1-16.

Kaiser, R., A. Maravall (2001), Measuring Business Cycles in Economic Time Series. Springer Verlag. 
King, R., S. Rebelo (1993), Low frequency filtering and real business cycles. Journal of Economic Dynamics and Control 17: 207-231.

McElroy, T. (2008), Matrix Formulas for Nonstationary ARIMA Signal Extraction. Econometric Theory 24: 988-1009.

Meyer, M., P. Winker (2005), Using HP Filtered Data for Econometric Analysis: Some Evidence from Monte Carlo Simulations. Allgemeines Statistisches Archiv 89: 303-320.

Pedersen, T.M. (2001), The Hodrick-Prescott filter, the Slutzky effect, and the distortionary effect of filters. Journal of Economic Dynamics and Control 25: 1081-1101.

Proietti, T. (2005), Forecasting and Signal Extraction with Misspecified Models. Journal of Forecasting 24: 539-556.

Proietti, T. (2007), Signal Extraction and Filtering by Linear Semiparametric Methods. Computational Statistics \& Data Analysis 52: 935-958.

Ravn, M.O., H. Uhlig (2002), On adjusting the Hodrick-Prescott filter for the frequency of observations. Review of Economic and Statistic 84: 371-380.

Sargent, T. (1987), Macroeconomic Theory. Academic Press.

Watson, M. (1986), Univariate Detrending Methods with Stochastic Trends. Journal of Monetary Economics 18: 49-75.

Whittle, P. (1983), Prediction and Regulation. Blackwell.

Prof. Dr. Gebhard Flaig, University of Munich, Schackstraße 4, 80539 Munich, Germany. gebhard.flaig@lrz.uni-muenchen.de 